recorded above has revealed the fact that although a demagnetising effect by damped, oscillating, H.F. magnetic fields was noted by Henry and afterwards studied by Rayleigh, Rutherford, Marconi and others, none of the investigators seems to have realised the relation of this theoretical work to practical demagnetisation.

Metallurgical Division,

$$
\text { C. W. Davis. }
$$

United States Bureau of Mines. Feb. 5 .

${ }^{1}$ Gottschalk, V. H., and Davis, C. W., "A Magnetic Material of High Coercive Force", NATURE, 132, 513, sept. 30, 1933.

\section{Variations in Interference Colours on Copper and Steel}

THE high values of the refractive index of copper and iron make it probable that only slight changes of colour would be observed on varying the angle of incidence of the light. With burnished metal surfaces, changes of larger magnitude than expected (giving $\mu=2.4$ for copper oxide and $\mu=1.8$ for iron oxide) were observed with angles of incidence up to $78^{\circ}$, together with strong scattering of the complimentary colours. At larger angles of incidence general reflection predominated, and all colour vanished except a slight effect due apparently to the metal itself.

Some curious results were observed on strips of metal cleaned with fine emery paper. The direction of the scratches was parallel to the length of the strip, and in the direction of increasing thickness of the oxide film. At angles of incidence varying from $70^{\circ}$ to $80^{\circ}$, the change in the second order colours was marked in the direction of the scratches. However, no change whatever (except increase in the generally reflected light) could be observed when the illumination was perpendicular to the lines of the scratches.

In this case the strips were mounted on a spectrometer table and the telescope replaced by a micro. scope. It then appeared that the colour of each filament remained unaltered, but that reflection occurred along a different line as the angle of inci. dence increased. Thus though the angle of incidence on the plane of the metal surface had varied widely, the angle of reflection from the coloured filaments in the surface had varied but slightly.

We wish to thank Abdel Hamid Effendi for the care he has taken in preparing the metal strips.

\section{F. H. Constable. \\ M. NAZIF. \\ H. ELDIN.}

Royal School of Engineering, Giza, Egypt.

\section{Rationalisation of Scientific Publication}

THE Publication Committee of the Society of Public Analysts and Other Analytical Chemists has discussed a reference (p. 358) in a leading article in NATURE of March 9 to the abstracts published by the Society in its journal, and I am instructed by the Committee to direct your attention to inaccuracies in the strictures contained in that article.
It is not true, as the article states, that the abstracts in The Analyst are merely later duplications or variations of abstracts already published by the Bureau of Chemical Abstracts. On the contrary, they are made directly from the original papers, and are frequently published before the abstracts issued by the Bureau. These points, however, are of minor significance as compared with the character of the abstracts themselves, which are constructed on completely different principles from those adopted by the Bureau. In the first place, all abstracts in The Analyst are given with sufficient detail to enable an analyst to try the method in his laboratory without having first to refer to the original paper. Secondly, every abstract is minutely scrutinised, first by a specialist in the subject, and then by the Publication Committee, and if the method is found to be unsound, or to be wanting in novelty, the abstract describing it is not published.

The principle adopted by the Bureau of Chemical Abstracts is different from this. Its object is to give the gist of the paper abstracted, leaving the reader to refer to the original paper if he wishes to obtain working details of any method. Doubtless this method of presenting abstracts saves space, but it cannot be claimed that it gives detailed working accounts of methods or affords any guidance to an analyst.

This Society is always willing to co-operate with other chemical societies, but attacks showing so little understanding of the objects and needs of a particular society will not tend to promote cooperation.

\section{Ainsworth Mitchell}

(Secretary and Editor).

Society of Public Analysts.

March 29.

Mr. Mrtchedu's letter in itself fully substantiates the statements made in the article. I do not think he is entitled to say that the article is in any way inaccurate. The substance of the charge is that the abstracts provided by the Society of Public Analysts in no way break fresh ground; they merely duplicate the effort which is expended by the British Chemical Abstracts, for example. Mr. Mitchell's letter shows clearly that the failure of the Society of Public Analysts to co-operate with the Bureau is due, first to a radical misconception as to what an abstract should provide, and second to the narrow specialist outlook which so largely frustrates efforts at cooperation.

With regard to the first point, it cannot be maintained that an abstract should provide the full working details required. If, as Mr. Mitchell appears to contend, an abstract is insufficient for the purpose of his Society, he is advocating essentially a policy of reprinting published information in whole or in part. This is in itself a lamentable source of duplicated effort and expenditure in the profession of chemistry, duo, as Mr. Mitchell's letter shows, to the specialist's insistence in putting sectional needs before the general interest.

The reference to the Society of Public Analysts, however, was only made by way of illustrating a point in the article, which covers a great deal of ground. It would not have been in keeping with the spirit of the article to have elaborated the point, even had there been any wish to do so.

The Writer of the Article. 